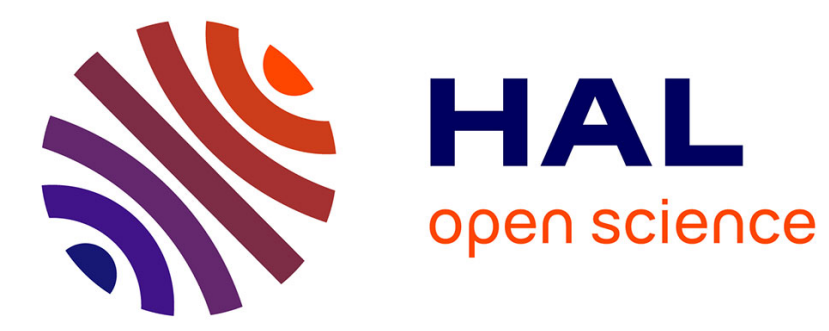

\title{
Temps de relaxation transversale du proton et réorientation du chloroforme dissous dans le sulfure de carbone
}

\author{
A. Briguet, J. Charrière, G. Tétu, J.C. Duplan, J. Delmau
}

\section{- To cite this version:}

A. Briguet, J. Charrière, G. Tétu, J.C. Duplan, J. Delmau. Temps de relaxation transversale du proton et réorientation du chloroforme dissous dans le sulfure de carbone. Journal de Physique Lettres, 1977, 38 (2), pp.73-76. 10.1051/jphyslet:0197700380207300 . jpa-00231329

HAL Id: jpa-00231329

https://hal.science/jpa-00231329

Submitted on 1 Jan 1977

HAL is a multi-disciplinary open access archive for the deposit and dissemination of scientific research documents, whether they are published or not. The documents may come from teaching and research institutions in France or abroad, or from public or private research centers.
L'archive ouverte pluridisciplinaire HAL, est destinée au dépôt et à la diffusion de documents scientifiques de niveau recherche, publiés ou non, émanant des établissements d'enseignement et de recherche français ou étrangers, des laboratoires publics ou privés. 


\title{
TEMPS DE RELAXATION TRANSVERSALE DU PROTON ET RÉORIENTATION DU CHLOROFORME DISSOUS DANS LE SULFURE DE CARBONE
}

\author{
A. BRiguet, J. CHARRIÈRE, G. TÉTU, J. C. DUPLAN et J. DELMAU \\ Université Claude-Bernard Lyon I, Laboratoire de Spectroscopie et de Luminescence, \\ 43, bd du 11-Novembre-1918, 69621 Villeurbanne, France
}

(Reçu le 5 octobre 1976, révisé le 6 décembre 1976, accepté le 16 décembre 1976)

\begin{abstract}
Résumé. - La mesure des temps de relaxation spin-spin et spin-milieu du proton de ${ }^{12} \mathrm{CHCl}_{3}$ et ${ }^{13} \mathrm{CHCl}_{3}$ montre que la réorientation du chloroforme dissous dans $\mathrm{CS}_{2}$ est inertielle et pratiquement isotrope.
\end{abstract}

Abstract. - Proton spin-spin and spin-lattice relaxation times for ${ }^{12} \mathrm{CHCl}_{3}$ and ${ }^{13} \mathrm{CHCl}_{3}$ diluted in $\mathrm{CS}_{2}$ show that molecular reorientation of chloroform is inertial and nearly isotropic.

1. Introduction. - La relaxation magnétique $\mathrm{nu}$ cléaire dans les systèmes de spins couplés est un sujet d'actualité $[1,2]$. Les travaux entrepris portent essentiellement sur la relaxation spin-milieu et conduisent à des informations sur la réorientation moléculaire. La présence de molécules isotopomères, en ${ }^{12} \mathrm{C}$ et ${ }^{13} \mathrm{C}$ par exemple, permet l'étude de la relaxation spinmilieu alors que les observations ne portent que sur un seul noyau, l'hydrogène en l'occurrence $[3,4,5]$. Cette note a pour objet de montrer comment on peut tirer parti de ce même effet isotopique lors d'une étude de la relaxation spin-spin dans un système $\mathbf{A X}$.

2. Partie expérimentale. - Nous avons mesuré les temps de relaxation spin-spin des raies protoniques d'un échantillon de chloroforme enrichi à $90 \%$ en carbone 13 et dissous à raison de $5 \times 10^{-2} \mathrm{M} / 1$ dans le sulfure de carbone. Etant donné la faible concentration en ${ }^{12} \mathrm{CHCl}_{3}$ de cet échantillon, pour plus de précision nous avons également effectué des mesures de $T_{1}$ et $T_{2}$ sur un échantillon de chloroforme naturel pris dans les mêmes conditions. Les expériences ont été effectuées à $30^{\circ} \mathrm{C}$ dans un champ de 2,3 Tesla, à l'aide d'un spectromètre à ondes continues utilisé en impulsions d'audiofréquence [6]. La méthode employée est celle du spin-locking [7] qui conduit à une mesure de $T_{1 \rho}$ égale à $T_{2}$ dans le cas des liquides et pour des valeurs suffisamment faibles du champ radiofréquence [8]. Afin d'obtenir des résultats probants à partir de raies de faible intensité nous avons mis en place un dispositif d'accumulation automatique des données [9]. Les résultats obtenus sont indiqués sur le tableau I. Une étude de $T_{2}$ sur des molécules isotopomères peut être plus intéressante

\section{TABLEAU I}

Mesures de $T_{1}$ et $T_{2}$ portant sur deux échantillons de chloroforme dissous à raison de $5 \times 10^{-2} \mathrm{M} / 1$ dans le sulfure de carbone à $30^{\circ} \mathrm{C}$. La différence des deux valeurs de $T_{20}$ observées provient d'une désoxygénation différente des deux échantillons. Toutefois il faut noter que la méthode d'obtention de $\boldsymbol{R}_{\mathrm{dd}}$ et $\boldsymbol{R}_{\mathrm{scal}}$ ne dépend pas du degré de désoxygénation étant donné que les impuretés affectent également toute mesure portant sur le même échantillon.

$$
\begin{array}{ll}
{ }^{1 \mathrm{er}} \text { échantillon } & \\
{ }^{13} \mathrm{CHCl}_{3} 90 \% & T_{20}=7,25 \pm 0,15 \mathrm{~s} \\
{ }^{12} \mathrm{CHCl}_{3} \quad 10 \% & T_{2 \mathrm{~s}}=6,36 \pm 0,05 \mathrm{~s} \\
\text { - } & R_{\mathrm{dd}}=(22,7 \pm 4,7) 10^{-3} \mathrm{~s}^{-1} \\
{ }^{2 \mathrm{e}} \text { échantillon } & \\
{ }^{13} \mathrm{CHCl}_{3} 1,1 \% & T_{10}=153 \pm 7 \mathrm{~s} \\
{ }^{12} \mathrm{CHCl}_{3} 98,9 \% & T_{20}=8 \pm 0,4 \mathrm{~s} \\
\text { (chloroforme } & R_{\text {scal }}=(118,5 \pm 7) 10^{-3} \mathrm{~s}^{-1} \\
\text { naturel) } &
\end{array}
$$

qu'une étude de $T_{1}$ car les temps de relaxation spinspin plus courts permettent de diminuer notablement la durée des mesures. D'autre part les résultats obtenus à partir de $T_{2}$ sont plus facilement exploitables que ceux obtenus à partir de $T_{1}$ car dans ce dernier cas les signaux n'évoluent pas selon une exponentielle unique [10]. Enfin la méthode de spin-locking est particulièrement aisée à mettre en œuvre en onde continue surtout lorsque l'intervalle spectral des raies 
est important devant l'amplitude du champ de radiofréquence utilisé [11].

3. Discussion. - Le calcul des temps de relaxation spin-spin des quatre raies d'un système $\mathrm{AX}$ montre que les deux raies d'un même noyau ont même temps de relaxation transversale et montre aussi que les fonctions de corrélation croisée entre les interactions aléatoires au niveau de $\mathrm{A}$ et de $\mathrm{X}$ n'interviennent pas [12].

En effet si l'on note

$$
R_{\mathrm{dd}}=\gamma_{\mathrm{A}}^{2} \gamma_{\mathrm{X}}^{2} \hbar^{2} r_{\mathrm{AX}}^{-6} \tau_{\mathrm{d}}
$$

le terme d'interaction dipolaire qui dépend du temps de corrélation $\tau_{\mathrm{d}}$ de la direction $\mathrm{AX}$, si de plus on introduit les densités spectrales du type $J_{\mathrm{AA}}(q)$, des fonctions de corrélation des interactions agissant au niveau $\mathrm{A}$ et conduisant à des transitions à $q$ quantum, et enfin si l'on utilise le formalisme de Redfield, le temps de relaxation spin-spin des deux raies $\mathrm{A}$ s'écrit :

$\frac{1}{T_{2 \mathrm{~A}}}=\frac{17}{20} R_{\mathrm{dd}}+\frac{1}{2 \hbar^{2}}\left(J_{\mathrm{AA}}(0)+2 J_{\mathrm{AA}}(1)+2 J_{\mathrm{XX}}(1)\right)$.

La relation donnant $T_{2 \mathrm{x}}$ est analogue puisqu'il suffit d'échanger les rôles de A et de X.

On remarque alors que l'existence du couplage introduit dans l'expression de l'inverse du temps de relaxation transversale des deux raies d'un même noyau un terme qui dépend uniquement de la relaxation spin-milieu de l'autre noyau. Ce terme est précisément la moitié de la vitesse de relaxation spinmilieu du second noyau en l'absence d'effet dipolaire direct intramoléculaire et peut être calculé dans l'hypothèse du champ local [13].

C'est ainsi que dans le cas des raies protoniques d'un mélange de molécules isotopomères ${ }^{12} \mathrm{CHCl}_{3}$ et ${ }^{13} \mathrm{CHCl}_{3}$, le temps de relaxation transversale $T_{2 \mathrm{~S}}$ des deux raies latérales $\left({ }^{13} \mathrm{CHCl}_{3}\right)$ s'exprime en fonction du temps de relaxation transversale $T_{20}$ de la raie centrale $\left({ }^{12} \mathrm{CHCl}_{3}\right)$, du terme d'interaction dipolaire $R_{\text {dd }}$ et de la contribution externe du carbone 13 , soit $T_{1 \mathrm{C}}^{-1},[1,11]$ :

$$
\frac{1}{T_{2 \mathrm{~S}}}=\frac{1}{T_{20}}+\frac{1}{2} \frac{1}{T_{1 \mathrm{C}}}+\frac{17}{20} R_{\mathrm{dd}} .
$$

Or dans le cas du carbone 13 du chloroforme les mécanismes externes restent faiblement efficaces devant l'interaction dipolaire $[14,15]$ et l'on peut facilement négliger (1/2) $T_{1 \mathrm{C}}^{-1}$ devant les deux autres termes du membre de droite de la relation (2). Ainsi la différence des temps de relaxation spin-spin protonique observés dans le premier échantillon (Tableau I) conduit à $R_{\mathrm{dd}}=(22,7 \pm 4,7) 10^{-3} \mathrm{~s}^{-1}$, soit pour temps de corrélation de réorientation de la direc- tion ${ }^{13} \mathrm{C}-{ }^{1} \mathrm{H}, \quad \tau_{\mathrm{d}}=1,1 \pm 0,2 \mathrm{ps}$, en prenant $r_{\mathrm{C}-\mathrm{H}}=1,1 \AA$ [16]. La liaison $\mathrm{C}-\mathrm{H}$ est dirigée suivant l'axe de symétrie moléculaire et par suite

$$
\tau_{\mathrm{d}}=\tau_{\theta \perp}=1 / 6 D_{\perp},
$$

soit $D_{\perp}=(1,5 \pm 0,3) 10^{11} \mathrm{~s}^{-1}, D_{\perp}$ est alors la constante de diffusion rotationnelle de réorientation $\mathrm{du}$ chloroforme autour d'un axe qui est perpendiculaire à son axe de symétrie et dont $\tau_{\theta \perp}$ représente le temps de corrélation.

Les trois noyaux quadrupolaires de chlore couplés scalairement avec le proton sont à l'origine d'un mécanisme très efficace de relaxation spin-spin $\mathrm{du}$ proton. Ce mécanisme contribue d'ailleurs très peu à sa relaxation spin-milieu car l'intervalle spectral entre les résonances du chlore et du proton est important devant l'inverse du temps de relaxation du chlore [17]. Par conséquent le temps de relaxation spin-spin $T_{20}$ du proton de ${ }^{12} \mathrm{CHCl}_{3}$ est nettement plus court que le temps de relaxation spin-milieu $T_{10}$ et la différence des inverses de ces deux grandeurs conduit à une contribution scalaire notée $R_{\text {scal }}$ :

$$
R_{\mathrm{scal}}=\frac{1}{T_{20}}-\frac{1}{T_{10}}
$$

L'expression de $R_{\text {scal }}$ est compliquée car il faut tenir compte de toutes les distributions d'isotopes ${ }^{35} \mathrm{Cl}$ et ${ }^{37} \mathrm{Cl}$ dans $\mathrm{CHCl}_{3}$. Une pondération sur les différentes formes isotopomères $\mathrm{du}$ chloroforme conduit à la relation pratique suivante :

$$
R_{\mathrm{scal}}=15,52 \pi^{2} J_{35}^{2} T_{1 \mathrm{~s}}\left({ }^{35} \mathrm{Cl}\right)
$$

où $J_{35}$ est la constante de couplage, exprimée en Hertz, du proton avec le chlore 35 dont l'orientation de spin dans le champ magnétique directeur est fonction aléatoire du temps avec $T_{1 \mathrm{~s}}\left({ }^{35} \mathrm{Cl}\right)$ pour temps de corrélation. Ce paramètre est égal au temps de relaxation spin-milieu du noyau quadrupolaire intéressé et pour établir la relation (4) nous avons admis que $T_{1}\left({ }^{37} \mathrm{Cl}\right) / T_{1}\left({ }^{35} \mathrm{Cl}\right)=1,64, \quad J_{35} / J_{37}=1,20 \quad[8]$. Grâce à cette formule la mesure de $R_{\text {scal }}$ permettra de déterminer $T_{1 \mathrm{~s}}\left({ }^{35} \mathrm{Cl}\right)$, et ensuite le temps de corrélation des directions $\mathrm{C}-\mathrm{Cl}$, à condition de connaître $J_{35}$. Malheureusement les valeurs trouvées dans la littérature pour la constante de couplage du proton et du chlore 35 présentent une dispersion importante. La valeur $J_{35}=5,25 \mathrm{~Hz}$, indiquée récemment par Dinesh et Rogers [18] dans une étude de la relaxation protonique du chloroforme pur à $25^{\circ} \mathrm{C}$, provient d'une estimation trop faible de $T_{1}\left({ }^{35} \mathrm{Cl}\right)=22,7 \mu \mathrm{s}$. L'emploi des résultats des mesures effectuées par Huntress $[15,19]$ nous amène à retenir une valeur de $\left(T_{1}{ }^{35} \mathrm{Cl}\right)=32,3 \mu \mathrm{s}$ à $25^{\circ} \mathrm{C}$ pour le chloroforme pur. Si on utilise alors les valeurs de $\left(T_{1}\right)_{\mathrm{H}}$ et $\left(T_{2}\right)_{\mathrm{H}}$ de Dinesh et Rogers, la relation (4) conduit à $J_{35}=4,25 \mathrm{~Hz}$. Compte tenu de ce résultat, la valeur déterminée pour $R_{\text {scal }}$ dans le second échantillon 
(Tableau I) donne alors, pour le chloroforme dissous à $5 \times 10^{-2} \mathrm{M} / 1$ dans le sulfure de carbone,

$$
T_{1 \mathrm{~s}}\left({ }^{35} \mathrm{Cl}\right)=42,8 \pm 4 \mu \mathrm{s}
$$

Le temps de relaxation du chlore s'exprime à son tour en fonction de sa constante de couplage quadrupolaire $Q$ et du temps de corrélation $\tau_{\theta \text { eff }}$ de réorientation de la liaison $\mathrm{C}-\mathrm{Cl}$.

$$
T_{1 \mathrm{~s}}\left({ }^{35} \mathrm{Cl}\right)^{-1}=\frac{2 \pi^{2}}{5} Q^{2} \tau_{\theta \mathrm{eff}}
$$

En prenant $Q=79 \mathrm{MHz}$ [20] on obtient

$$
\tau_{\theta \text { eff }}=0,95 \pm 0,1 \mathrm{ps}
$$

On notera au passage que ce moyen de détermination est le seul capable de donner $\tau_{\theta \text { eff }}$ dans un échantillon très dilué où toute mesure directe sur la relaxation du chlore est rendue très difficile du fait de sa faible sensibilité.

Dans le modèle de diffusion rotationnelle anisotrope [19], le temps de corrélation effectif $\tau_{\theta \text { eff }}$ de la liaison $\mathrm{C}-\mathrm{Cl}$ est fonction de l'angle $\theta$ des deux liaisons $\mathrm{C}-\mathrm{H}$ et $\mathrm{C}-\mathrm{Cl}$ ainsi que des constantes de diffusion de réorientation moléculaire autour de l'axe de symétrie $\left(D_{/ /}\right)$et perpendiculaire à celuici $\left(D_{\perp}\right)$ :

$$
\begin{aligned}
\tau_{\theta \mathrm{eff}}=\frac{\left(3 \cos ^{2} \theta-1\right)^{2}}{24 D_{\perp}}+\frac{3 \sin ^{2} \theta \cos ^{2} \theta}{5 D_{\perp}+D_{\|}}+ \\
+\frac{3 \sin ^{4} \theta}{4\left(2 D_{\perp}+4 D_{/ /}\right)}
\end{aligned}
$$

La géométrie de la molécule $\mathrm{CHCl}_{3}$ [16] donne $\theta=107^{\circ} 57$. Utilisons alors

$$
D_{\perp}=(1,5 \pm 0,3) 10^{11} \mathrm{~s}^{-1},
$$

nous obtenons :

soit

$$
D_{/ /}=(2,09 \pm 0,1) 10^{11} \mathrm{~s}^{-1},
$$

$$
\tau_{\theta / /}=1 / 6 D_{/ /}=0,8 \pm 0,1 \mathrm{ps}
$$

Le résultat $\tau_{\theta / /}<\tau_{\theta \perp}$ est physiquement plausible et justifie a posteriori l'adoption de la valeur $4,25 \mathrm{~Hz}$ pour $J_{35}$. D'autre part la valeur $\tau_{\theta \perp}=1,1 \pm 0,2 \mathrm{ps}$ que nous avons obtenue par relaxation magnétique du proton de $\mathrm{CHCl}_{3}$ dilué dans $\mathrm{CS}_{2}$ peut être comparée au temps de relaxation diélectrique $\tau_{\mathrm{D}}=2,7 \mathrm{ps}$ mesuré dans des conditions analogues à $20^{\circ} \mathrm{C}$ [21].

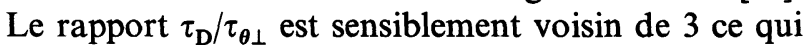
indique que la diffusion de réorientation a probablement un caractère brownien.

Les valeurs très voisines des temps de corrélation des axes principaux de $\mathrm{CHCl}_{3}\left(\tau_{\theta \perp}=1,1 \mathrm{ps}\right.$ et $\tau_{\theta / /}=0,8 \mathrm{ps}$ ) montrent que la réorientation du chloroforme dilué dans le sulfure de carbone est sensiblement isotrope. Par ailleurs la question se pose de savoir si la réorientation moléculaire est de type inertiel. Dans cette dernière hypothèse, si on applique au chloroforme le modèle inertiel de Steele [22, 23], on trouve pour temps de corrélation de réorientation $\tau_{\mathrm{f}} \simeq 0,51$ ps à $30^{\circ} \mathrm{C}$. Le rapport entre les temps de corrélation expérimentaux et le temps de corrélation $\mathrm{du}$ modèle inertiel est donc voisin de 2 (comme cela a été observé pour le chlorure de méthyle mais au contraire de ce qui a été observé pour les autres halogénures du méthane [24]). La réorientation du chloroforme dilué présente donc un caractère inertiel prononcé.

L'ensemble de ces résultats portant sur du chloroforme dilué est à rapprocher de ceux de Huntress [19] ; cet auteur a mis en évidence le caractère anisotrope de la réorientation $\left(\tau_{\theta \perp}=1,8 \mathrm{ps}, \tau_{\theta / /}=0,92 \mathrm{ps}\right)$ ainsi que l'existence d'effets inertiels déjà non négligeables pour le chloroforme pur à $20^{\circ} \mathrm{C}$. Il semble que les interactions entre les moments dipolaires moléculaires jouent un rôle important dans la réorientation : ils expliqueraient l'anisotropie et le caractère brownien des mouvements. La diminution de la concentration des moments dipolaires réduirait leurs interactions mutuelles permettant ainsi des mouvements isotropes et plus libres donc inertiels. Aussi serait-il intéressant d'étendre la méthode que nous proposons à des études à concentration variable dans différents solvants.

Nous tenons à remercier le rapporteur pour ses remarques pertinentes et ses suggestions qui ont permis une discussion complète de nos résultats.

\section{Bibliographie}

[1] Vold, R. R. and Vold, R. L., J. Chem. Phys. 64 (1976) 320.

[2] Wang, C. H. and Grant, D. M., J. Chem. Phys. 64 (1976) 1522.

[3] Ozawa, H., Arata, Y. and Fujiwara, S., J. Chem. Phys. 57 (1972) 1613

[4] Heatley, F., J. Chem. Soc. Faraday Trans. II 70 (1974) 148.

[5] Briguet, A., Duplan, J. C. and Delmau, J., J. Mol. Phys. 29 (1975) 837.

[6] Briguet, A., Culty, J. C., Duplan, J. C. and Delmau, J., J. Phys. E : Sci. Instrum. 7 (1974) 791.
[7] Solomon, I., C. R. Hebd. Séan. Acad. Sci. 248 (1959) 92.

[8] Strange, J. H. and Morgan, R. E., J. Phys. C : Solid State Phys. 3 (1970) 1999.

[9] Lapray, C., Briguet, A., Duplan, J. C. and Delmau, J., J. Magn. Res. 23 (1976) 129.

[10] Briguet, A., Duplan, J. C. and Erbeia, A., J. Physique 32 (1971) 23.

[11] Freeman, R. and Wittekoek, S., J. Magn. Res. 1 (1969) 238.

[12] Freeman, R., Wittekoek, S. and ERnst, R. R., J. Chem. Phys. 52 (1970) 1529. 
[13] Kumar, A. and Rao, B. D. N., J. Magn. Res. 8 (1972) 1.

[14] Farrar, T. C., Druck, S. J., Shoup, R. R. and Becker, E. D., J. Amer. Chem. Soc. 94 (1972) 699.

[15] Shoup, R. R. and FarraR, T. C., J. Magn. Res. 7 (1972) 48

[16] Jen, M. and LiDe, D. R., J. Chem. Phys. 36 (1962) 2525.

[17] Ce n'est pas le cas lorsqu'on opère à champ faible. Cf. WiNTER, J. M., C. R. Hebd. Séan. Acad. Sci. 249 (1959) 1346.

[18] Dinesh and Rogers, M. T., Chem. Phys. Lett. 12 (1971) 352.

[19] Huntress, W. T., J. Phys. Chem. 73 (1969) 103.
[20] Hooper, H. O. and Bray, P. J., J. Chem. Phys. 33 (1960) 334.

[21] Whiffen, D. H., Trans. Faraday Soc. 46 (1950) 130.

[22] Miller, C. R. and Gordon, S. L., J. Chem. Phys. 53 (1970) 3531.

[23] Monz, W., Steele, W. and Dixon, J., J. Chem. Phys. 38 (1963) 2418.

[24] Brown, P. M., Krishna, N. R. and Gordon, S. L., J. Magn. Res. 20 (1975) 540. 